\title{
Osteoimmunology: a new area of rheumatology research
}

\author{
Peter E Lipsky
}

Growing awareness of the close links between the immune and skeletal systems has stimulated development of a new field: osteoimmunology (Walsh MC et al. [2006], Annu Rev Immunol 24: 33-63; Takayanagi H [2007], Nat Rev Immunol 7: 292-304). This discipline generally focuses on three main topics. The first relates to developmental issues, especially the potential role of bone in the development of the immunohemopoietic system; less attention has been devoted to the reciprocal relationship, because initial skeletogenesis seems to occur independently of the immune system. The second focus relates to the influence of the immune system on the skeletal system, and vice versa, during homeostasis, whereas the third area of focus concerns the role of the immune system in bone pathology and the potential involvement of bone in immune system dysfunction.

Several important observations provided the underpinnings of osteoimmunology. First, despite its superficially inert appearance, bone is rapidly and continuously remodeled at a high rate by bone-forming osteoblasts and boneresorbing osteoclasts. The latter differentiate from myeloid cells of the immune system, which demonstrates the intimate relationship between bone and the immune system. Second, and closely related to the first point, the functions of osteoclasts and osteoblasts are positively and negatively influenced by a variety of cytokines and surface molecules, including tumor necrosis factor (TNF) and several interleukins, produced by cells of the immune system. Immune system cells can, therefore, influence the balance between bone loss and bone matrix formation.

Third, cells residing in the bone marrow cavity, including osteoblasts and bone marrow stromal cells, provide essential factors that influence the survival, self-renewal capacity and pluripotency of hemopoietic stem cells. Interplay between osteoblasts and hemopoietic stem cells involves a number of ligand-receptor interactions, including $\mathrm{N}$-cadherin- $\beta$-catenin, jagged-notch 1 , angiopoietin 1-tyrosine kinase receptor 2 , the ...osteo-

immunology

encompasses

the analysis of

developmental,

homeostatic

and pathologic

consequences

of the

interactions

of the immune

and skeletal

systems

PE Lipsky is the Editor-in-Chief of Nature Clinical Practice Rheumatology.

Competing interests

The author declared no competing interests.

www.nature.com/clinicalpractice doi:10.1038/ncprheum0764
CXC chemokine ligand-receptor pair CXCL12CXCR4, as well as interactions involving Wnt signaling. The bone marrow microenvironment also provides survival niches for antibodysecreting plasma cells and memory $\mathrm{T}$ and $B$ cells. These niches are primarily associated with bone marrow stromal cells and involve several cytokines, chemokines and adhesion molecules. In addition, osteoclasts produce large amounts of B-cell activating factor (also known as TNF ligand superfamily member $13 \mathrm{~B}$, or B-lymphocyte stimulator), which can enhance the survival of plasma cells and other B-lineage cells.

Finally, pathologic damage to bone is mediated by the interaction of immune system cells-in particular, by soluble products derived from such cells-and bone cells, with the development of bone erosion in rheumatoid arthritis (RA) providing a good example of such damage. Bone damage in RA is mediated by increased osteoclast differentiation and decreased osteoblast function at sites of inflammation; proinflammatory cytokines, such as TNF, seem to be crucial for both processes. TNF increases the expression by osteoblasts of receptor activator of nuclear factor $\mathrm{\kappa B}$ ligand, and can thereby increase the local differentiation of bone-resorbing osteoclasts; this cytokine also increases production of Dickkopf 1 , which inhibits Wnt signaling, and thereby decreases the bone-synthetic activity of osteoblasts (Diarra D et al. [2007], Nat Med 13: 156-163). Consequently, blocking TNF in RA exerts a powerful inhibitory effect on the progression of bone damage, by suppressing osteoclast function and stimulating bone synthesis by osteoblasts.

The new discipline of osteoimmunology encompasses the analysis of developmental, homeostatic and pathologic consequences of interactions between the immune and skeletal systems. Understanding these complex interactions should provide new insights into the functional regulation of both systems, and also uncover new targets for therapeutic intervention. 